\title{
Índice de resistencia de la arteria cerebral anterior en el neonato muy prematuro
}

\section{Resistance index of the anterior cerebral artery in the very premature neonate}

\author{
Elia María Ortiz Borda ${ }^{1,2, a}$, Violeta Alicia Nolberto Sifuentes ${ }^{3, b}$ \\ ${ }^{1}$ Servicio de Neonatologia, Hospital Nacional Edgardo Rebagliati Martins, Seguro Social de Salud EsSalud. Lima, Perú. \\ ${ }^{2}$ Facultad de Medicina, Universidad Nacional Mayor de San Marcos. Lima, Perú. \\ ${ }^{3}$ Facultad de Ciencias Matemáticas, Universidad Nacional Mayor de San Marcos. Lima, Perú. \\ ${ }^{a}$ Neonatóloga, ORCID: 0000-0001-5668-5772 \\ ${ }^{\mathrm{b}}$ Estadística, ORCID: https://orcid.org/0000-0003-1577-4298
}

An Fac med. 2020;81(4):416-9. / DOI: https://doi.org/10.15381/anales.v81i4.16732

Correspondencia:

Elia Ortiz Borda

emortizb2002@yahoo.es

Recibido: 21 de agosto 2020

Aprobado: 15 de diciembre 2020

Publicación en línea: 15 de febrero 2021

Conflictos de interés: Las autoras declara no tener conflictos de interés.

Fuente de financiamiento:

Autofinanciado

Citar como: Ortiz E, Nolberto V. Indice de resistencia de la arteria cerebral anterior en el neonato muy prematuro. An Fac med. 2020;81(4):416-9. DOl: https://doi.org/10.15381/anales. v81i4.16732

\section{Resumen}

Introducción. El índice de resistencia (IR) de la arteria cerebral anterior (ACA) permite evaluar el flujo sanguíneo y se utiliza para determinar el bienestar fetal. Objetivo. Determinar el flujo de la arteria cerebral anterior en muy prematuros durante las primeras 72 horas de vida. Métodos. Se revisaron historias clínicas de muy prematuros atendidos en la Unidad de Cuidados Intensivos Neonatales del Hospital Nacional Edgardo Rebagliati Martins (HNERM), EsSalud, Lima-Perú, entre los años 2011-2019. Resultados. Se revisaron 56 historias clínicas que reportaron el índice de resistencia de la arteria cerebral anterior en muy prematuros del HNERM. Los valores promedio del IR de la ACA fueron: 0,695 a las 24 horas; 0,69 a las 48 horas; y 0,667 a las 72 horas. El flujo sistólico promedio de la arteria cerebral anterior a las 48 horas de vida fue similar al de la vida intrauterina; a las 72 horas, el flujo diastólico promedio fue menor. Conclusiones. En muy prematuros atendidos en la Unidad de Cuidados Intensivos Neonatales del HNHRM, los valores promedio del IR de la ACA fueron: 0,695 a las 24 horas; 0,69 a las 48 horas; y 0,667 a las 72 horas.

Palabras clave: Arteria Cerebral Anterior; Recién Nacido Prematuro; Ultrasonografía Doppler Transcraneal (fuente: DeCS BIREME).

\section{Abstract}

Introduction. The anterior cerebral artery (ACA) resistance index (IR) is used to assess blood flow and is used to determine fetal well-being. Objective. To determine the flow of the anterior cerebral artery in very preterm infants during the first 72 hours of life. Methods. Medical records of very preterm infants treated in the Neonatal Intensive Care Unit of Edgardo Rebagliati Martins National Hospital (HNERM), EsSalud, Lima-Peru, between the years 2011-2019 were reviewed. Results. 56 medical records were reviewed that reported the index of resistance of the anterior cerebral artery in very premature infants of the HNERM. The mean values of the IR of the ACA were: 0,695 at 24 hours; 0,69 at 48 hours; and 0,667 at 72 hours. The mean systolic flow of the cerebral artery prior to 48 hours of life was similar to that of intrauterine life; at 72 hours, the mean diastolic flow was lower. Conclusions. In very preterm infants treated in the Neonatal Intensive Care Unit of the HNHRM, the average RI values of the ACA were: 0,695 at 24 hours; 0,69 at 48 hours; and 0,667 at 72 hours.

Keywords: Anterior Cerebral Artery; Infant, Premature; Ultrasonography, Doppler, Transcranial (source: MeSH NLM). 


\section{INTRODUCCIÓN}

Los muy prematuros (menores de 32 semanas) son hemodinamicamente inestables, con riesgo de hemorragias cerebrales, leucomalacia e insuficiencia cardiaca. Por la presencia del ductus arterioso en un corazón inmaduro y debido a su tamaño, labilidad y riesgo de infecciones, es difícil la realización de procedimientos invasivos así como su movilización ${ }^{(1-4)}$. En ellos, conocer el comportamiento del índice de resistencia de la arteria cerebral anterior (ACA) podría ser de utilidad para la detección precoz de alteraciones hemodinámicas ${ }^{(1,5)}$.

La ecografía doppler transcraneal permite la monitorización no invasiva, no ionizante y sin desplazamiento del paciente. Es de gran ayuda en los muy prematuros, quienes cuentan con fontanelas que facilitan el estudio ecográfico (5,6); así, la fontanela anterior, que es la más amplia, permite obtener imágenes nítidas en el corte sagital, y con una leve angulación del traductor, se obtiene el recorrido de la arteria cerebral anterior desde su segmento A2 ${ }^{(7,8)}$. La arteria cerebral anterior tiene cinco segmentos: $A 1$ segmento horizontal, A2 ascendente, A3 rodilla, A4 peri orbital o pericallosal, y A5 peri marginal o terminal.

El índice de resistencia (IR) se obtiene utilizando el doppler pulsado mediante las velocidades sistólicas y diastólicas, lo que permite conocer el flujo sanguíneo ${ }^{(7,8)}$. Existe poca diferencia del valor del IR en las diferentes edades gestacionales $(4,5,6)$; asimismo, podría estar influenciado por la presencia del ductus arterioso y la concentración de $\mathrm{CO}_{2}$ (hipercarbia) $)^{(5,7,9,10)}$.

Existen pocos reportes del comportamiento del flujo de la arteria cerebral anterior en el muy prematuro durante los primeros días de vida ${ }^{(11,12)}$. El presente estudio tuvo como objetivo determinar el flujo de la arteria cerebral anterior en muy prematuros durante las primeras 72 horas de vida, utilizando el plano sagital a nivel de A2. Se considera que las primeras 72 horas de vida son el periodo de mayor inestabilidad hemodinámica en el muy prematuro, debido a que en dicho periodo la presión de la arteria pulmonar desciende más del 50\% debido a mecanismos de adaptación a la vida extrauterina, con el mayor riesgo de descompensación hemodinámica por inversión del flujo del lado sistémico al lado pulmonar del corazón, por la persistencia del ductus arterioso ${ }^{(14)}$. Su conocimiento es fundamental para la detección de cambios en el estado hemodinámico, lo que permitiría un tratamiento más oportuno, disminuyendo las complicaciones de patologías que cursen con alteración de la resistencia periférica $(13,15,16,18)$.

\section{MÉTODOS}

Se realizó un estudio observacional descriptivo. Se revisaron las historias clínicas de los muy prematuros atendidos en la Unidad de Cuidados Intensivos Neonatales del Hospital Nacional Edgardo Rebagliati Martins, EsSalud, entre los años 2011 a 2019.

Se utilizaron las historias que contaban con el valor del índice de resistencia de la arteria cerebral anterior en las primeras 72 horas de vida. Se registraron los valores del IR de la arteria cerebral anterior por edad gestacional y peso, a las 24, 48 y 72 horas de vida. El ecógrafo utilizado fue Sonoace 300 con transductor convexo pediátrico multifrecuencia de 5 a $9 \mathrm{MHz}$. Las medidas del IR la realizaron tres neonatólogos del Servicio de Neonatología del Hospital Nacional Edgardo Rebagliati Martins, quienes utilizaron la técnica estandarizada para la toma de las medidas, como parte de la atención habitual a los recién nacidos muy prematuros. Se utilizó el plano sagital, segmento ascendente
A2, a $5-10 \mathrm{~mm}$ antes de $\mathrm{A} 3$ (rodilla) con ángulo de insonación de $20^{\circ}$.

El estudio tuvo la aprobación del Comité de Ética del Hospital Nacional Edgardo Rebagliati EsSalud. Se mantuvo la confidencialidad de los datos obtenidos.

\section{RESULTADOS}

Se encontraron 56 historias clínicas con registros del IR de la arteria cerebral anterior. Las edades gestacionales se encontraron en el rango de 24 a 32 semanas, con una media de 28,7 semanas; el peso promedio de los neonatos fue de 1249 gramos, con un rango de 525 gramos a 2025 gramos. El 56,8\% fueron menores de 29 semanas. Se encontraron valores promedio del IR de la ACA $24 \mathrm{~h}$ 0,695, ACA 48h 0,69, ACA 72h 0,667, la desviación estándar y rangos se muestran en la tabla 1.

Los valores promedio de flujos sistólicos fueron de $22,7 \mathrm{~mL} / \mathrm{min}$ (DS 7,4) a las 24h; 26,5 mL/min (DS 8,2) a las 48h; 28,4 $\mathrm{mL} / \mathrm{min}(D S 7,2)$ a las $72 \mathrm{~h}$; y los valores promedio de flujo diastólico de $6,9 \mathrm{~mL} /$ min (DS 3,4) a las 24h; $8,9 \mathrm{~mL} / \mathrm{min}$ (DS 3,8 ) a las 48 horas; y 9,2 mL/min (DS 2,6) a las 72 horas, tal como se muestra en la figura 1. Las distribuciones fueron normales para las velocidades flujo sistólico y diastólico de 24 y 48 horas, no así en la velocidad diastólica a las 72 horas de vida.

En la tabla 2 se muestra los valores comparativos respecto a los hallazgos de Govaert ${ }^{(13)}, \mathrm{y}$ Liu ${ }^{(19)}$ (valores intrautero).

Tabla 1. Índice de resistencia de la arteria cerebral anterior (IR-ACA) a las $24 \mathrm{~h}, 48 \mathrm{~h}$ y $72 \mathrm{~h}$, en muy prematuros. Unidad de Cuidados Intensivos Neonatales, Hospital Nacional Edgardo Rebagliati Martins, 2011 - 2019.

\begin{tabular}{lccc} 
& IR ACA 24h & IR ACA 48h & IR ACA 72h \\
\hline Media & 0,695 & 0,690 & 0,667 \\
\hline Mediana & 0,690 & 0,700 & 0,680 \\
\hline Desviación estándar & 0,099 & 0,074 & 0,112 \\
\hline Rango & 0,50 & 0,03 & 0,81 \\
\hline Mínimo & 0,49 & 0,56 & 0,20 \\
\hline Máximo & 0,99 & 0,83 & 0,81 \\
\hline
\end{tabular}



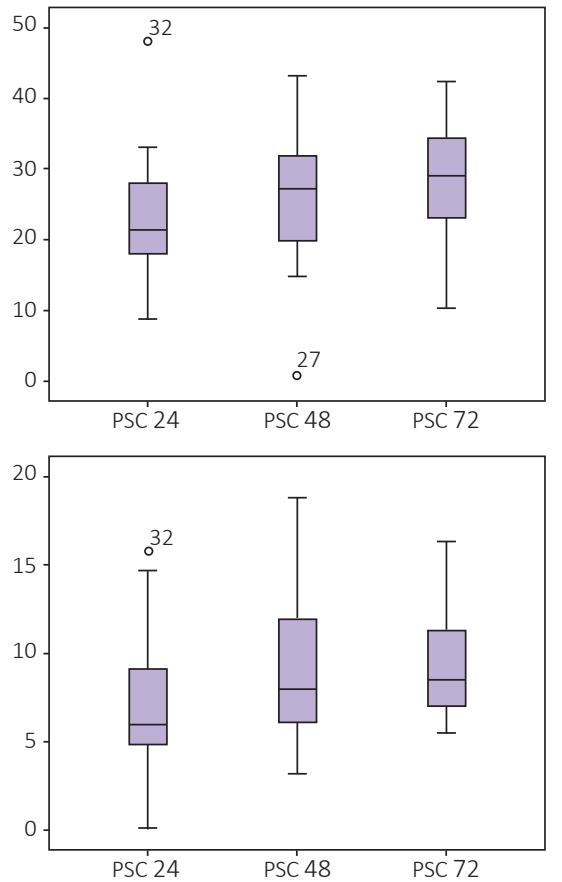

Figura 1. Flujo sistólico en $\mathrm{mL} / \mathrm{min}$ (izquierda) y flujo diastólico en $\mathrm{mL} / \mathrm{min}$ (derecha) de la arteria cerebral anterior a las 24,48 y 72 horas de vida en muy prematuros. Unidad de Cuidados Intensivos Neonatales, Hospital Nacional Edgardo Rebagliati Martins, 2011 - 2019.

\section{DISCUSIÓN}

El flujo sistólico encontrado en los 56 prematuros evaluados fue mayor según la edad gestacional, y tuvo una distribución normal. El IR a las $24 \mathrm{~h}, 48 \mathrm{~h}$, y 72 h, fue menor a lo reportado previamente en valores intrauterinos ${ }^{(19)}$, probablemente debido al secuestro diastólico que puede suceder con el ductus arterial persistente ${ }^{(13)}$ y al aumento de la resistencia periférica que se produce en la sepsis, así como la enterocolitis ${ }^{(10)}$, que son las tres complicaciones más frecuentes en estos pacientes y causas de alteración hemodinámica cuya incidencia es mayor a partir de las 72 horas de vida ${ }^{(10,13)}$.

Los valores del índice de resistencia de la arteria cerebral anterior fueron menores a lo reportado por Govaert ${ }^{(13)}$ y similares a los valores intrauterinos encontrados por Liu ${ }^{(19)}$, quien a diferencia del estudio de Govaert ${ }^{(13)}$ (plano coronal), utilizó el plano sagital para identificar la arteria cerebral anterior, por ser el más

Tabla 2. Índice de resistencia de la arteria cerebral anterior obtenidas en muy prematuros del Hospital Rebagliati 2011-2019, en comparación con los estudios de Govaert ${ }^{(13)}$, Liu ${ }^{(19)}$.

\begin{tabular}{cccc} 
Horas de vida & Govaert $^{(13)}$ & Liu $^{(19)}$ & Obtenidas \\
\hline $24 \mathrm{~h}^{(19)}$ & $0,79(0,73-0,86)$ & $0,72(+/-0,06)$ & $0,695(+/-0,03)$ \\
\hline $48 \mathrm{~h}$ & 0,81 & & 0,69 \\
\hline $72 \mathrm{~h}$ & 0,84 & 0,667 \\
\hline
\end{tabular}

usado en la práctica clínica, por ser más sencillo para obtener las imágenes, lo que también fue descrito por Kumar ${ }^{(15)}$. La utilidad del presente estudio es dar a conocer la variabilidad que existe en los valores del índice de resistencia de la arteria cerebral anterior que se produce a las 72 horas de vida, donde algunos muy prematuros tendrán flujo invertido por el ductus, y otros no, de acuerdo a las presiones pulmonares y tamaño ductal. El índice de resistencia de la arteria cerebral anterior podría ser una ayuda para identificar precozmente a estos dos grupos de pacientes.

Las limitaciones de nuestra investigación se encuentran relacionadas a que el estudio fue retrospectivo, donde no es posible controlar variables intervinientes Si bien los planos de corte y las técnicas son estandarizadas, el procedimiento realizado por tres médicos podría intervenir en los resultados. De igual manera, no se ha considerado la presencia de patologías concomitantes en los neonatos.

Nuestros hallazgos permiten conocer valores iniciales del índice de resistencia de la arteria cerebral anterior, usando el plano sagital que es el más útil en la práctica clínica neonatal. Estos valores son estrechos y la variación es marcada a las 72 horas de vida. El índice de resistencia de la arteria cerebral anterior podría ser útil para identificar precozmente a los neonatos que cursan con alteraciones hemodinámicas.

\section{REFERENCIAS BIBLIOGRÁFICAS}

1. Sociedad Española de Cuidados Intensivos Pediátricos [Internet]. Mirás A, Montero R, Valencia J. Neuroecografia en el paciente critico pediatrico. Grupo de trabajo ecografía [Fecha de acceso: 6 de abril 2020]. Disponible en: https://secip.com/wp-content/uploads/2018/09/5NEUROECOGRAF\%C3\%8DA-EN-EL-PACIENTEPEDI\%C3\%81TRICO-CR\%C3\%8DTICO.pdf
2. Heck S, Schindler T, Smyth J, Lui K, Meriki N, Welsh A. Evaluation of neonatal regional cerebral perfusion using power Doppler and the index fractional moving blood volume. Neonatology. 2012;101(4):254-9. DOI: 10.1159/000334648

3. Wu TW, Azhibekov T, Seri I. Transitional hemodynamics in preterm neonates: Clinical relevance. Pediatr Neonatol. 2016;57(1):7-18. DOI: 10.1016/j. pedneo.2015.07.002

4. D'andrea A, Conte M, Scarafile R, Riegler L, Cocchia R, Pezzullo E, et al. Transcranial Doppler Ultrasound: Physical Principles and Principal Applications in Neurocritical Care Unit. J Cardiovasc Echogr. 2016;26(2):28-41. DOI: 10.4103/22114122.183746

5. Wu TW, Tamrazi B, Soleymani S, Seri I, Noori S. Hemodynamic Changes During Rewarming Phase of Whole-Body Hypothermia Therapy in Neonates with Hypoxic-Ischemic Encephalopathy. J Pediatr. 2018;197:68-74.e2. DOI: 10.1016/j. jpeds.2018.01.067

6. Forster DE, Koumoundouros E, Saxton V, Fedai G, Holberton J. Cerebral blood flow velocities and cerebrovascular resistance in normal-term neonates in the first 72 hours. J Paediatr Child Health. 2018;54(1):61-8. DOI: https://doi.org/10.1111/ jpc.13663

7. Barrientos-Guerra JD, Flores-Silva F, Cantú-Brito C Chiquete E. Evaluation of Cerebral Hemodynamics with Color-Coded Duplex Sonography: Normative Values with Correction of Insonation Angles. J Stroke Cerebrovasc Dis. 2020;29(3):104595. DOI: DOI: 10.1016/j.jstrokecerebrovasdis.2019.104595

8. Bertini G, Coviello C, Gozzini E, Bianconi T, Bresci C, Leonardi V, et al. Change of Cerebral Oxygenation during Surfactant Treatment in Preterm Infants: "LISA" versus "InSurE" Procedures. Neuropediatrics. 2017;48(2):98-103.

9. Zhang W, Xie S, Han D, Huang J, Ou-Yang C, Lu J. Effects of relative low minute ventilation on cerebral haemodynamics in infants undergoing ventricular septal defect repair. Cardiol Young. 2020;30(2):205-212. DOI: 10.1017/ S1047951119003135

10. El-Dib M, Soul JS. Monitoring and management of brain hemodynamics and oxygenation. In: Handbook of Clinical Neurology. Elsevier B.V.; 2019. p. 295-314. DOI: 10.1016//B978-0-44464029-1.00014-X

11. Serrano I. Utilidad clínica del indice de resistencia cerebral mediante ecografía doppler en el recién nacido. Influencia del DAP hemodinámicamente significativo en el índice de resistencia cerebral [Tesis Maestria]. Universidad de Zaragoza; 2014.

12. Elmfors AF, Sandgren T, Ford K, Rosenberg J, Ringertz $\mathrm{H}$, Barth RA, et al. Normal values of the resistivity index of the pericallosal artery with and without compression of the anterior fontanelle. Pediatr Radiol. 2019;49(5):646-651. DOI: 10.1007/ s00247-019-04347-y 
13. Ecury-Goossen G, Raets M, Camfferman F, Vos $\mathrm{RHJ}$, van Rosmalen J, Reiss IKM, et al. Resistive indices of cerebral arteries in very preterm infants: values throughout stay in the neonatal intensive care unit and impact of patent ductus arteriosus. Pediatr Radiol. 2016;46:1291-300. DOI: 10.1007/ s00247-016-3615-x

14. Ngo S, Profit J, Gould JB, Lee HC. Trends in Patent Ductus Arteriosus Diagnosis and Management for Very Low Birth Weight Infants. Pediatrics. 2017;139(4):e20162390. DOI: 10.1542/ peds.2016-2390

15. Kumar AS, Chandrasekaran A, Asokan R, Gopinathan K. Prognostic Value of Resistive Index in
Neonates with Hypoxic Ischemic Encephalopathy. Indian Pediatr. 2016;53(12):1079-82.

16. Benitz WE. Patent Ductus Arteriosus in Preterm Infants. Pediatrics. 2015;137(1):e20153730. DOI: 10.1542/j.peds.2015-3730

17. Furuichi $\mathrm{K}$, Ishikawa $\mathrm{A}$, Uwabe $\mathrm{C}$, Makishima $\mathrm{H}$, Yamada S, Takakuwa T. Variations of the Circle of Willis at the End of the Human Embryonic Period. Anat Rec. 2018;301(8):1312-9. DOI: 10.1002/ ar.23794

18. Bertuetti R, Gritti P, Pelosi P, Robba C. How to use cerebral ultrasound in the ICU. Minerva Anestesiol. 2020;86(3):327-340. DOI: 10.23736/ S0375-9393.19.13852-7
19. Liu Y, Xiao Y, Zeng S, Tan Y, Zhou J, Zhao B, et al. Blood flow parameters in fetal anterior cerebral artery during the second and third trimester of gestation. J Cent South Univ (Medical Sci. 2018;43(9):994-9. DOI: 10.11817/j.issn.16727347.2018.09.0

20. Ecury-Goossen GM, Raets MMA, Camfferman FA, Vos RHJ, van Rosmalen J, Reiss IKM, et al. Resistive indices of cerebral arteries in very preterm infants: values throughout stay in the neonatal intensive care unit and impact of patent ductus arteriosus. Pediatr Radiol. 2016;46(9):1291-300. DOI: 10.1007/s00247-016-3615-x 